\title{
Pediatric Sacral Nerve Stimulator Explanation due to Complications or Cure: A Survival Analysis
}

Adam J Rensing, Konrad M Szymanski, Sally Dunn, Shelly King, Mark P Cain, and Benjamin M Whittam

Division of Pediatric Urology

Department of Urology, Indiana University School of Medicine Indianapolis, Indiana 46202

Corresponding Author:

Benjamin M Whittam, MD, MS

705 Riley Hospital Drive, Suite 4230

Division of Pediatric Urology

Indianapolis, IN 46202

Phone: 001-317-948-8556

Fax: 001-317-944-7481

Email: bwhittam@iupui.edu 


\section{Introduction:}

Historically, there have been few treatment options for children with severe, refractory bladder and bowel dysfunction (BBD). Sacral neuromodulation (SNM) continues to show promising results in this challenging pediatric population with recalcitrant lower urinary tract symptoms. At our institution, we have begun offering explantation to those with persistent improvement after $>6$ months of having device turned off. We hypothesized that 1.) SNM explantation for cure increases with extended follow-up, and 2.) those explanted for cure would have improved symptoms and quality of life when compared to those explanted for complication.

\section{Materials \& Methods:}

We retrospectively reviewed all consecutive patients $<18$ years old who underwent SNM placements at our institution (2012-2017). We excluded those without the second stage procedure. Reasons for device explantation were categorized as: cure (resolution of symptoms with the device turned off for at least 6 months), or a complication (e.g. infection, need for MRI, or pain). Non-parametric tests and survival analysis were used for analysis to account for differential follow-up time. Of those explanted, surveys were electronically sent to assess BBD severity, and overall quality of life.

Results:

Of 67 children who underwent a first stage procedure, $62(92.5 \%)$ underwent a second stage procedure. 61 met inclusion criteria $(68.9 \%$ female, $29.5 \%$ with previous filum section, median age at implantation 10.3 years old). During follow-up (median 2.3 years), 12 patients (19.7\%) had the SNM exchanged/revised due to lead fracture/breakage and return of urinary symptoms. To date, 50 patients remain with their SNM implanted, and 11 have been explanted. Adjusting for follow-up time, the risk of explantation was $6.5 \%$ at 2 years $(2.2 \%$ for cure, $4.3 \%$ for complications) (Figure 1). Explantation increased to $24.5 \%$ at 3 years $(16.5 \%$ for cure, $8.0 \%$ for complications) and $40.4 \%$ at 4 years (32.4\% for cure, $8.0 \%$ for complications). Questionnaires were collected on patients post explant (median 2.2 years), with improvement in those explanted for cure compared to complication (Figure 2).

\section{Discussion:}

SNM explantation for cure is a novel concept previously not described in the literature. Limitations of this study include the relatively small numbers, and lack of objective data in the cohort that remains with SNM device implanted.

\section{Conclusion:}

SNM is a safe, viable option for the pediatric patient with refractory bladder dysfunction. Furthermore, SNM explantation for cure is an option with increasing likelihood after two years. 


\section{Introduction:}

Within the practice of pediatric urology, lower urinary tract dysfunction (LUTD), bowel dysfunction, and, more generally, bladder and bowel dysfunction (BBD) are frequent diagnoses of varying severity. Children often present with symptoms of increased or decreased urinary frequency, urgency, nocturia, hesitancy, straining, weak stream, intermittency, urinary incontinence, and/or dysuria. These symptoms correlate with urodynamic findings of detrusor overactivity, detrusor sphincter discoordination, and an otherwise normal neurologic history and physical exam [1].

First line therapy in this population consists of behavioral modification, or urotherapy [2, 3]. Also, constipation needs to concurrently be addressed in order to rule out the effects of rectal distension upon bladder function, given the previously described pelvic organ "crosssensitization" [4]. Previous authors have described "cross-sensitization" as the "transmission of noxious stimuli from a directly affected pelvic organ to an adjacent normal structure." For those with persistent, refractory symptoms, techniques of biofeedback, focused physical therapy, anticholinergics, alpha blockers, and noninvasive, transcutaneous neurostimulation may be considered [2].

In 2004, SNM (a therapeutic option consisting of permanent electrical stimulation of the sacral nerves at the S3 level using an implantable pulse generator device ) was first reported as a potential option in the pediatric patient population [5]. In this study, children (mainly with spina bifida) were randomized to SNM implantation or control (mainly intermittent catheterization, anticholinergics, and bulking agent for continence or reflux), with some evidence of clinical improvement, however, urodynamic differences were not statistically significant. Later, larger 
studies affirmed an improvement in treating neurologically normal children's refractory bladder and bowel symptoms as well as quality of life [6-10]. Limited data exists as to the "life expectancy" of the SNM in the pediatric population. Dwyer et al described explantation rates in children (38 devices at median 2.36 years, 12 for complete symptom resolution). We are unaware of any other data in the pediatric or adult SNM literature that describes voluntary explant for "cure." Within the pediatric realm, families that have experienced a profound relief of symptoms following SNM implantation seek prognostic data as to when this device may stop having a therapeutic benefit and be explanted, and/or require further surgery.

We hypothesized that 1.) SNM explantation for cure and complications increases with extended follow-up, and 2.) those explanted for cure would have improved symptoms and quality of life when compared to those explanted for complication.

\section{Materials \& Methods:}

After obtaining approval from our institutional review board, we retrospectively reviewed all children ( $<18$ years of age) treated at our institution for refractory bladder dysfunction with implantation of a SNM device between November 2012 and September 2017. Those considered refractory to conservative measures had not improved after a prolonged trial of behavioral modification, dietary modification, biofeedback/focused physical therapy, aggressive treatment of concomitant constipation, and pharmacologic therapy with anticholinergics that lasted for 2 years or more. Only after all of these more conservative options were exhausted was SNM offered. In addition, a spinal MRI was obtained on all patients to exclude a previously unrecognized, occult spinal dysraphism (OSD) by screening for a low lying conus and/or fatty/thickened filum terminale [11]. Those with any signs of a tethered cord were referred to 
neurosurgery for possible filum section/detethering. However, we included in our analysis those with persistent symptoms, despite filum section, and treated with SNM. We excluded patients who did not go on to have the second stage procedure performed due to less than $50 \%$ improvement of symptoms following 2-week trial. The most recent follow up was recorded of all patients in the cohort.

Operative Procedure:

Children were treated with implantation of an InterStim $I^{\circledR}$ device (Medtronic, Inc., Minneapolis, MN, USA). The first stage was performed in an operating room under general anesthesia with the patient in the prone position, using a tined lead placed adjacent to the S3 nerve root. This is confirmed fluoroscopically, as well as with confirmation of the bellows response and plantar flexion of the great toe with stimulation on the ipsilateral side. Patients and their families then use an external generator for a 2-week trial period. During this trial period, patients' families $\backslash$ are diligently called by our support staff to obtain a symptom diary, and provide as needed adjustments to their SNM device. Those that describe a significant (>50\%) improvement in their bladder dysfunction symptoms are given the option to proceed to the second stage, involving placement of an internalized generator in the subcutaneous space in the contralateral superior gluteal fold.

Following implantation, we keep in close contact with all families with regular follow up. For those with complete response after at least one year after implantation, we offer a trial of turning the device off to check for symptom resolution. Subsequently, in those with complete success with the device off for $>6$ months, we offered explantation. We originally had planned for 1 year, but some families requested removal earlier, given persistent improvement. 


\section{Outcomes}

We retrospectively collected demographic and SNM surgery data. For those who underwent device explantation, the indication for explantation was noted. We categorized indications as cure (resolution of symptoms with device off for $>6$ months) or complication (infection, need for an MRI for further neurosurgical evaluation, or refractory pain in the general area). To account for differential follow-up times, we performed a Kaplan-Meier survival analysis to graphically examine the impact of explantation for these two reasons while adjusting for differential followup time. In order to assess long-term symptom control, we contacted the patients that have been explanted, and sent them electronic questionnaires that included the Vancouver Symptom Score for Dysfunctional Elimination Syndrome (VSSDES) and the Pediatric Urinary Incontinence quality of life questionnaire (PinQ) to objectively quantify the degree of BBD, and the effect of their current level of bladder dysfunction upon their wellbeing, respectively $[12,13]$. We also collected PedsQL scores to evaluate overall health-related quality of life currently [14].

\section{Results:}

Of the 67 children who underwent a first stage procedure, $62(92.5 \%)$ underwent a second stage procedure. After excluding 1 child that was lost to follow-up, 61 met inclusion criteria ( $68.9 \%$ female, $29.5 \%$ status post filum section, median 10.3 years old). Median follow-up time was 2.3 years. Children who did and did not undergo a second stage procedure were similar in age at implantation and gender $(p>=0.11)$ (Table 1). During follow-up, 12 patients (19.4\%) that went on to have the second stage procedure had the SNM exchanged/revised due to lead fracture/breakage and return of urinary symptoms. Subsequently, we compared those explanted (11) to those that remained with the device in place (50) (Table 2). The follow-up 
time was significantly longer in the explanted group, due to the criteria required (described above) for explantation to be offered. Otherwise, all other demographics were similar.

\section{Explantation Analysis}

We performed a survival analysis of the 61 children meeting inclusion criteria to evaluate the likelihood and time course of explantation for different indications. To date, 50 patients remain with their SNM implanted and 11 have been explanted ( 8 for cure, 3 for complications). No devices in this cohort were explanted for lack of response/effect. We found that most explantations were performed after 2 years of follow-up (Figure 1). Adjusting for differential follow-up time, the risk of explantation was $6.5 \%$ at 2 years $(2.2 \%$ for cure, $4.3 \%$ for complications, Table 3). Explantation increased to $24.5 \%$ at 3 years (16.5\% for cure, $8.0 \%$ for complications) and $40.4 \%$ at 4 years (32.4\% for cure, $8.0 \%$ for complications).

Measuring Quality of Life:

We then looked to the post explantation questionnaires. These were administered a median of 1.6 and 2.5 years following explant in the cure and complication cohorts, respectively. Of the 11 patients with a SNM explant, we were able to contact 8 (unable to contact 3 explanted for cure). For the VSSDES, we found a median score of 9 in those explanted for cure (max/worst score of 56). This compared to 21 in the three explanted for complication. The PINQ scores also were markedly different. Of those explanted for cure, the median PINQ score was 12 , as compared to 59 in the complication group (max/worst score possible of 80 ). Lastly, the general quality of life was also documented in those explanted for complication and cure using the PedsQL questionnaire (max score of 100 considered maximum quality of life). Again, those explanted 
for cure demonstrated a marked difference with a median score of 83.3 , as compared to 46.7 in those explanted for complication.

\section{Discussion:}

This retrospective analysis of pediatric patients with severe, refractory bladder symptoms provides some needed prognostic information for providers as well as parents. Although previous studies have demonstrated the efficacy of SNM in this population $[6,7,10,15,16]$, our data would further suggest that explantation of the device after consistent symptom improvement is not only possible, but clinically effective, and durable.

Lloyd et al. discuss their experience with explantation in the adult population [17]. We could not find mention of this anywhere else in the adult literature. In their study, 90 patients underwent device removal after implantation. Of these, removal occurred for MRI (21 patients, $23 \%$ ), lack of efficacy (51 patients, $57 \%$ ), infection (10 patients, $11 \%$ ), and pain (8 patients, $9 \%$ ).

Interestingly, one third (7) of the MRI group reported preoperative, suboptimal symptom control, and only $10 \%$ (2) sought device replacement following explant. Of the remaining 19 who had the device removed prior to an MRI, 7 sought pharmacologic therapy, 3 intermittent catheterization, 2 Botulinum toxin, one local urologic care, one cystectomy/ileal conduit, one died, and 4 were lost to follow up.

Our findings in the pediatric population would suggest that there is a sustained benefit after therapy. Unfortunately, many questions remain to be answered as to primary mechanism by which SNM works at all. The theoretical advantage of electrical neurostimulation use in childhood compared to adults is the increased neuroplasticity of central and peripheral nervous 
systems with the potential for altered long-term outcomes [16]. With increasing evidence in adult LUTS literature, these adult conditions (overactive bladder, urgency, etc.) may be inherent and longstanding [16, 18-21]. This could explain why, in the adult population, LUTS seem to invariably return after explant [17]. One wonders, if this effect is sustained, perhaps we could alter or eliminate future, adult LUTS with initial treatment in childhood.

Dwyer et al. provide some valuable insights in their 10-year experience in the pediatric population [6]. Interestingly, despite 99/105 (94\%) of children experiencing improvement of at least 1 symptom following implantation, they noted reoperations in as many as 59/105 (56 \%), mostly due to device malfunction. Our data suggested a reoperation rate of $12 / 61(19.7 \%)$. They noted explantation in 38/105 (35\%), mainly for complete symptom resolution at a median of 2.4 years after implantation. It was unclear whether a trial period with device turned off was performed, and how those explanted in their cohort fared following explant. As noted above, we had an explant rate of $11 / 61(18.0 \%)$ after a median follow up of 2.22 years. Of note, unlike Dwyer et al, we have always employed the 2 stage technique.

The strength of our study is its robust, pediatric cohort with intermediate follow-up and a subset of post-explant cured patients. To our knowledge, this durable response has not been previously described in the pediatric neurourologic literature. In addition, we used multiple measures (PEDS QL, Vancouver questionnaire, PINQ) to objectively measure changes in quality of life and LUTS.

Weaknesses of this study include the inherent weaknesses of many retrospective studies. An unrecognized, confounding variable could influence who was offered and consented to device explant. Despite being offered explant, families/parents can choose whether to remain with their device, and if ever to explant the device. In addition, our population is small and somewhat 
heterogeneous with a subset previously treated with filum section. Also, survey results were sometimes difficult to obtain, and the most difficult to contact (3/11 explanted patients) were, perhaps, paradoxically, those that had the greatest improvement following implantation. Lastly, follow up remains short for those explanted, and theoretically, symptoms could return.

\section{Conclusion:}

SNM is a viable option for the pediatric patient with refractory bladder dysfunction after all other less invasive options have been exhausted. Over $90 \%$ of patients selected for SNM undergo permanent device placement. SNM has low explantation rates for complications, a high likelihood of continued benefit from the device, with a progressively increasing chance for explantation for cure beginning 2 years after implantation. Further study of this population will be interesting to see if this benefit has continued durability.

\section{Acknowledgements:}

All named authors have made meaningful contributions toward this work, and have agreed to their inclusion. This material is original, and not been published nor submitted elsewhere.

\section{Funding Statement:}

The authors received no funding from an external source.

\section{Conflicts of Interest:}


There were no conflicts of interest identified for any of the named authors regarding this work.

\section{References}

1. Austin, P.F., et al., The standardization of terminology of lower urinary tract function in children and adolescents: update report from the Standardization Committee of the International Children's Continence Society. J Urol, 2014. 191(6): p. 1863-1865 e13.

2. Santos, J.D., R.I. Lopes, and M.A. Koyle, Bladder and bowel dysfunction in children: An update on the diagnosis and treatment of a common, but underdiagnosed pediatric problem. Canadian Urological Association Journal, 2017. 11(1-2Suppl1): p. S64-S72.

3. Austin, P.F., et al., The standardization of terminology of lower urinary tract function in children and adolescents: Update report from the standardization committee of the International Children's Continence Society. Neurourol Urodyn, 2016. 35(4): p. 471-81.

4. Malykhina, A.P., K.E. Brodie, and D.T. Wilcox, Genitourinary and gastrointestinal comorbidities in children: The role of neural circuits in regulation of visceral function. J Pediatr Urol, 2017. 13(2): p. 177-182.

5. Guys, J.M., et al., Sacral neuromodulation for neurogenic bladder dysfunction in children. J Urol, 2004. 172(4 Pt 2): p. 1673-6.

6. Dwyer, M.E., et al., Sacral neuromodulation for the dysfunctional elimination syndrome: a 10-year single-center experience with 105 consecutive children. Urology, 2014. 84(4): p. 911-7. 
7. Mason, M.D., et al., Prospective Evaluation of Sacral Neuromodulation in Children: Outcomes and Urodynamic Predictors of Success. J Urol, 2016. 195(4 Pt 2): p. 1239-44.

8. Schober, M.S., et al., Sacral Nerve Stimulation for Pediatric Lower Urinary Tract Dysfunction: Development of a Standardized Pathway with Objective Urodynamic Outcomes. J Urol, 2015. 194(6): p. 1721-6.

9. Lu, P.L., et al., Sacral nerve stimulation for constipation and fecal incontinence in children: Long-term outcomes, patient benefit, and parent satisfaction. Neurogastroenterol Motil, 2017.

10. Sulkowski, J.P., et al., Sacral nerve stimulation: a promising therapy for fecal and urinary incontinence and constipation in children. J Pediatr Surg, 2015. 50(10): p. 1644-7.

11. Tuite, G.F., et al., Evaluation and management of tethered cord syndrome in occult spinal dysraphism: Recommendations from the international children's continence society. Neurourol Urodyn, 2018. 37(3): p. 890-903.

12. Afshar, K., et al., Development of a symptom score for dysfunctional elimination syndrome. J Urol, 2009. 182(4 Suppl): p. 1939-43.

13. Bower, W.F., et al., PinQ: a valid, reliable and reproducible quality-of-life measure in children with bladder dysfunction. J Pediatr Urol, 2006. 2(3): p. 185-9.

14. Varni, J.W., M. Seid, and C.A. Rode, The PedsQL: measurement model for the pediatric quality of life inventory. Med Care, 1999. 37(2): p. 126-39.

15. Haddad, M., et al., Sacral neuromodulation in children with urinary and fecal incontinence: a multicenter, open label, randomized, crossover study. J Urol, 2010. 184(2): p. 696-701. 
16. Wright, A.J. and M. Haddad, Electroneurostimulation for the management of bladder bowel dysfunction in childhood. Eur J Paediatr Neurol, 2017. 21(1): p. 67-74.

17. Lloyd, J.C., et al., Removal of Sacral Nerve Stimulation Devices for Magnetic Resonance Imaging: What Happens Next? Neuromodulation, 2017.

18. Fitzgerald, M.P., et al., Childhood urinary symptoms predict adult overactive bladder symptoms. J Urol, 2006. 175(3 Pt 1): p. 989-93.

19. Kuh, D., L. Cardozo, and R. Hardy, Urinary incontinence in middle aged women: childhood enuresis and other lifetime risk factors in a British prospective cohort. J Epidemiol Community Health, 1999. 53(8): p. 453-8.

20. Heron, J., et al., Trajectories of urinary incontinence in childhood and bladder and bowel symptoms in adolescence: prospective cohort study. BMJ Open, 2017. 7(3): p. e014238.

21. Doiron, R.C., et al., Childhood bladder and bowel dysfunction predicts irritable bowel syndrome phenotype in adult interstitial cystitis/bladder pain syndrome patients. Can Urol Assoc J, 2017. 11(8): p. 255-259. 


\section{Table 1:}

\begin{tabular}{|c|c|c|c|}
\hline & Did not complete 2 nd stage (5) & $\begin{array}{l}\text { Completed } 2^{\text {nd }} \text { Stage } \\
(62)\end{array}$ & $P$ value \\
\hline Female & $3(60.0 \%)$ & 43 (69.3\%) & $0.65^{*}$ \\
\hline $\begin{array}{l}\text { Median age at } \\
\text { implantation } \\
\left.\left(1 \text { st } Q, 3^{\text {rd }} Q\right)\right)\end{array}$ & $\begin{array}{l}8.1 \text { years } \\
(7.9,8.2)\end{array}$ & $\begin{array}{r}10.1 \text { years } \\
(7.8,12)\end{array}$ & $0.09 * *$ \\
\hline $\begin{array}{l}\text { Median Follow up } \\
\left.\text { (1st } Q, 3^{\text {rd }} Q\right)\end{array}$ & $\begin{array}{r}0.54 \text { years } \\
(0.4,1.8)\end{array}$ & $\begin{array}{r}2.22 \text { years } \\
(1,3)\end{array}$ & $0.14^{* *}$ \\
\hline Previous Filum Section & $1(20 \%)$ & $18(29 \%)$ & 0.99* \\
\hline
\end{tabular}

*Using Fischer's exact test.

**Using simple T-test. 


\section{Table 2:}

\begin{tabular}{|l|r|r|r|}
\hline & Device Remains (51) & Device Explanted (11) & P Value \\
\hline Female & $34(66.7 \%)$ & $9(81.8 \%)$ & 0.48 \\
\hline Previous Filum Section & $17(33.3 \%)$ & $1(9.1 \%)$ & 0.15 \\
\hline Revision Rate & $8(15.7 \%)$ & $4(36.4 \%)$ & 0.20 \\
\hline $\begin{array}{l}\text { Median age at Implant } \\
\left(1^{\text {st }} Q, 3^{\text {rd }} Q\right)\end{array}$ & $9.9(7.7,12.1))$ & $10.4(7.8,11.6)$ & 0.72 \\
\hline $\begin{array}{l}\text { Median Follow up }\left(1^{\text {st }}\right. \\
\left.\text { Q, } 3^{\text {rd }} Q\right)\end{array}$ & 2.1 years $(1.0,2.8)$ & 3.2 years $(2.4,3.5)$ & 0.001 \\
\hline $\begin{array}{l}\text { Median time to Explant } \\
\left(1^{\text {st }} Q, 3^{\text {rd }} Q\right)\end{array}$ & & & 2.6 years $(1.4,3.0)$ \\
\hline
\end{tabular}

(Excluded those without $2^{\text {nd }}$ stage.) 


\section{Table 3.}

\begin{tabular}{|l|l|l|l|l|}
\hline $\begin{array}{l}\text { Time after SNM } \\
\text { implantation } \\
\text { (years) }\end{array}$ & $\begin{array}{l}\text { Number of } \\
\text { patients } \\
\text { followed in } \\
\text { this time- } \\
\text { frame }\end{array}$ & $\begin{array}{l}\text { Remain with } \\
\text { SNM }\end{array}$ & $\begin{array}{l}\text { Explanted for } \\
\text { cure }\end{array}$ & $\begin{array}{l}\text { Explanted for } \\
\text { complication }\end{array}$ \\
\hline 1 year & $60(100.0 \%)$ & $59(98.2 \%)$ & $0(0.0 \%)$ & $1(1.8 \%)$ \\
\hline 2 years & $47(78.3 \%)$ & $45(93.5 \%)$ & $1(2.2 \%)$ & $1(4.3 \%)$ \\
\hline 3 years & $35(58.3 \%)$ & $30(75.5 \%)$ & $4(16.5 \%)$ & $1(8.0 \%)$ \\
\hline 4 years & $12(20.0 \%)$ & $10(59.6 \%)$ & $2(32.4 \%)$ & $0(8.0 \%)$ \\
\hline 5 years & $5(8.3 \%)$ & $4(39.7 \%)$ & $1(52.3 \%)$ & $0(8.0 \%)$ \\
\hline
\end{tabular}

Risk of sacral neuromodulation device explantation due to resolution of symptoms or complications. Note: percentages are based on survival analysis calculations adjusting for decreasing number of patients remaining in follow-up over the course of the study. 
Figure 1: Kaplan-Meier Survival Curve for all Implanted SNM Devices



Figure. Incidence of sacral neuromodulation device explantation due to complications or resolution of symptoms. 
Figure 2: Explant Surveys for those Explanted for Cure and Complication

\begin{tabular}{|l|l|l|l|l|}
\hline \multirow{2}{*}{} & \multicolumn{2}{l|}{ Explanted for Cure } & \multicolumn{2}{l|}{ Explanted for Complication } \\
\cline { 2 - 5 } & Median Score & $\%$ Abnormal & Median Score & \% Abnormal \\
\hline $\begin{array}{l}\text { Dysfunctional Elimination } \\
\text { Score }\end{array}$ & $9^{\wedge}$ & $40 \% *$ & 21 & $66 \% *$ \\
\hline PIN Q Score & 12 & $100 \%$ mild** & 59 & $33 \%$ moderate. \\
severe**
\end{tabular}

Figure. ^ Raw scores in the cure cohort were: 5, 8, 9, 15, 16

*Based upon cutoff score of 11 suggested by Afsar, et al. ${ }^{2}$

${ }^{* *}$ Based upon Thibodeau et al's proposed mild $=<21$, moderate $=21-50, \mathrm{~m}$ and severe $>50^{3}$ 